\title{
The difference between individuals and social groups in multidirectional movement
}

\author{
Yanghui Hu, Jun Zhang*, Weiguo Song* \\ State Key Laboratory of Fire Science \\ University of Science and Technology of China \\ No.96, JinZhai Road Baohe District, Hefei, China \\ huyang1@mail.ustc.edu.cn; junz@ustc.edu.cn; wgsong@ustc.edu.cn
}

\begin{abstract}
Social groups exhibit some degree of social cohesion that is more than a simple collection or aggregation of individuals. The study on behaviours of social groups is essential to gain a deeper understanding on pedestrian dynamics. In this paper, the crowd movement among individuals and social groups are studied by performing a series of multidirectional pedestrian flow experiments under laboratory condition. Interestingly, we found that the speed of individual is not always higher than that of social groups. Pedestrians try to use different strategies to arrive their destination. With different strategies, their speed and movement time show different properties. These findings may provide basis for facility design and evacuation plan.
\end{abstract}

Keywords: Social group; Individual; Pedestrian dynamic; Speed; Movement time

\section{Introduction}

The studies on human safety and pedestrian dynamics are growing rapidly in recent years. The comprehensive understanding on pedestrian dynamics is essential for crowd management and facility design. The major component of the crowd is social groups [1-4]. However, most of previous studies focused on individuals but only a few works consider the influence of social groups in a crowd on pedestrian dynamics.

Speed is one of the basic parameters for describing pedestrian dynamics. Many researchers have pointed out that the velocity of social groups is smaller than that of individuals. Moussaid et al. took an observation and found that the walking speed of pedestrians decreased linearly with the increasing of group size [3]. Zanlungo et al. concluded that three-person groups walked slower than two-person groups, and the latter walked slower than individual pedestrians [5]. Li et al. observed that the average velocity of group members $(\mathrm{v}=1.072 \mathrm{~m} / \mathrm{s})$ was smaller than that of individuals $(\mathrm{v}=1.15 \mathrm{~m} / \mathrm{s})$ from a field study [6]. Gorrini et al. focused the impact of different densities on pedestrian velocity [7,8]. They found that the walking speed of social groups was slower than that of individuals in both low and high densities. Zhao et al. found that the walking speed of individuals was significantly faster than that of dyads both in low and high densities in a subway station [9]. However, from control experiments Wagnlid et al. found that the speed of male dyads was larger than that of male individuals [10]. From these studies, it seems that there is no consensus on the speed difference between social groups and individuals.

Besides, under the same situations individuals and social groups may choose different walking path due to their different flexibility and synergy. Gorrini et al found that the walk path of individual was $4 \%$ longer than that of social group through observation [8]. Besides, they noticed that the walk path of adult individuals was longer than that of adult dyads and elderly dyads [11]. Zhao et al. found no difference of the walking path between individuals and social groups in low densities from an observation in a subway station in Beijing. However, under high density situations dyads trended to walk in a more straight way compared to individual [9].

Furthermore, most of these results are obtained from uni- or bidirectional flow. In daily life, we often encounter with multidirectional flow, for example, in the atrium of shopping malls, train stations 
and subway stations and so on. In these locations, pedestrians come from different directions and go to different destinations. To investigate the difference between social groups and individuals, we carried out experiments to study the difference between social groups and individuals under multidirectional flow.

\section{Experiment Setup}

The experiment was carried out in November 2017 in the University of Science and Technology of China, Anhui, China. The experiment scenario is a circle with the radius of $5 \mathrm{~m}$ which is used to form multidirectional flows [11]. Fig. 1 shows the sketch of the experiment scenario and a snapshot of the experiment (the red pedestrians represent the individuals and the blue pedestrians represent the social groups). In this paper, we only consider pairs as social group since the proportion of pair is larger than $50 \%$ of crowd in most case [1-4]. In this paper, a special relationship that is the strong paired relationship between pairs was adopted. For each pair, they were asked to keep in physical touch (arm in arm) with each other in the experiment. At the beginning of the experiment, participants stood on the border of the circle (cross standing between social groups and individuals in theory) and individuals separated different groups uniformly. The destination of each pedestrian is the opposite circle arc through the centre of the circle. Pedestrians were asked to reach their destination as quickly as possible in each run with the same instruction. During the movement, they can freely choose their preferred manner to avoid collision with other participants to arrive at their destinations. In total, 72 participants took part in the experiment. They are all undergraduate students from University of Science and Technology of China and 18-24 years old. Six runs were performed with different numbers of participants. In each run, the numbers of social groups and individuals are the same (except scenario 01 where the number of individual is one more than that of social groups). The ratio of boys to girls is close to one and thus we ignored the impact of gender and age on the results. The details of the experiment are shown in Table 1.

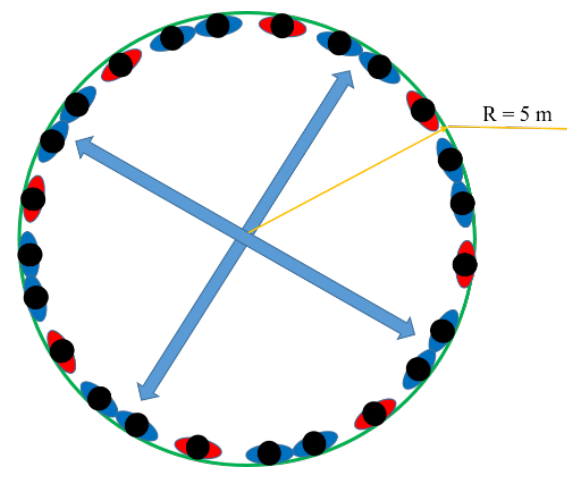

(a)

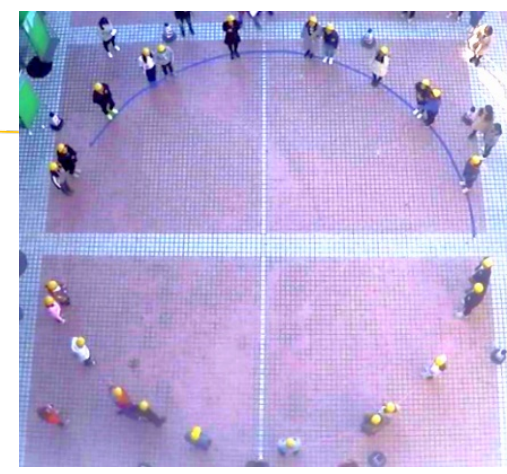

(b)

Fig. 1: (a) The schematic illustration of the scenario and (b) a snapshot of the experiment.

Table 1. The details of the experiment.

\begin{tabular}{|c|c|c|c|}
\hline Scenario & Pedestrians number & Number of social groups & Number of individuals \\
\hline 01 & 13 & 4 & 5 \\
\hline 02 & 24 & 8 & 8 \\
\hline 03 & 36 & 12 & 12 \\
\hline 04 & 48 & 16 & 16 \\
\hline 05 & 60 & 20 & 20 \\
\hline 06 & 72 & 24 & 24 \\
\hline
\end{tabular}

Two cameras were fixed on the 5th floor of a building to record the whole experiment. The trajectories of pedestrians were automatically extracted from video recordings using the software PeTrack 
[12]. Finally, the trajectories of the whole experiment were corrected manually. The frame rate of the trajectories data corresponds to $25 \mathrm{fps}$.

\section{Trajectories}

Based on high precision tracking of PeTrack, the characteristics of pedestrians including trajectories, velocity and density can be determined. Due to the head swing of the pedestrian, the trajectories display the periodic change and cannot show the real position of the body. We smooth the trajectories according to the step frequency which is about $2 \mathrm{~Hz}$. We use $1 \mathrm{~s}$ as the smoothing window and calculate the average value as the $\mathrm{x}$ and $\mathrm{y}$ coordinate. Results show that it can eliminate the effect of head swing well. We draw the trajectories of pedestrians for run 1 to run 6 in Fig 2. The red lines represent the individuals and the blue lines represent the trajectories of social groups.
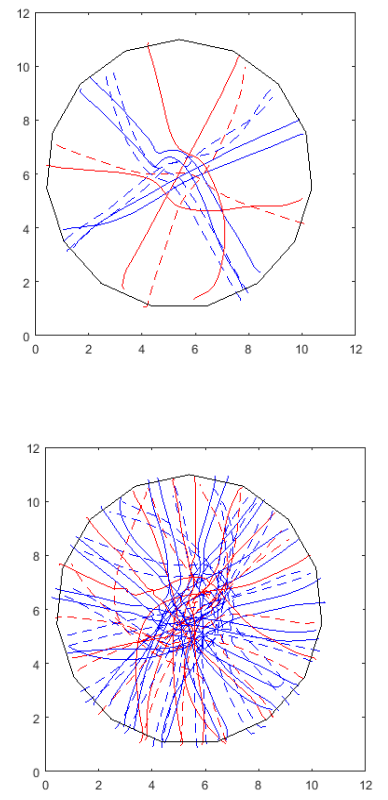

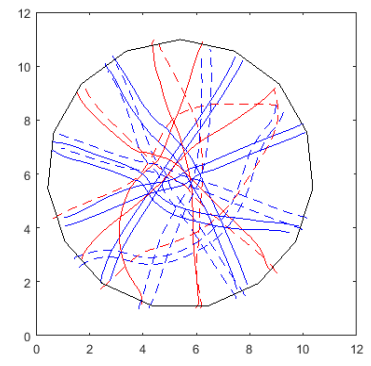

(a)

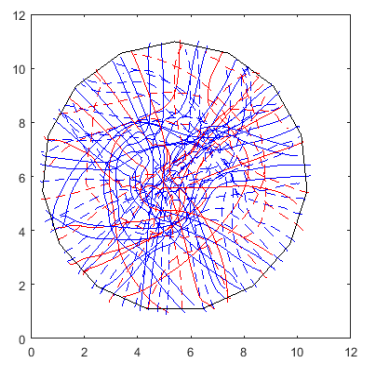

(b)
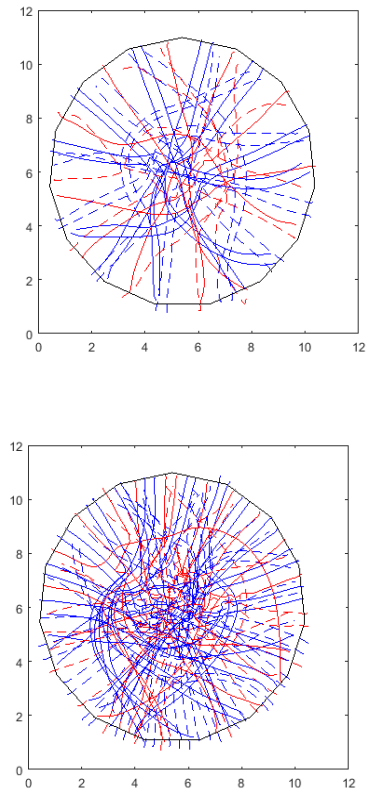

Fig. 2. The trajectories of (a) scenario 01 to 03 (b) scenario 04 to 06.

From the figures, we can see that social group move together and they keep a relative small interpersonal distance. In low density, the walk path is more orderliness and few detour phenomenon appears. Pedestrians can get through the central area straight. However, with the increase of participants, the trajectories of pedestrians becomes complicated gradually. The trajectories in central area (area 2 which will be explained below) is seriously crossed and obvious detour behaviour appears in the experiment. Serious congestion occurred in the central area and pedestrian are stuck in the area and cannot move quickly. In order to reach the target quickly, some pedestrians made a detour.

\section{Data Analysis Method}

The instantaneous velocity $\bar{v}_{l}(t)$ is defined as below:

$$
\bar{v}_{l}(t)=\frac{\sqrt{\left[x_{i}\left(t+\Delta t^{\prime} / 2\right)-x_{i}\left(t-\Delta t^{\prime} / 2\right)\right]^{2}+\left[y_{i}\left(t+\Delta t^{\prime} / 2\right)-y_{i}\left(t-\Delta t^{\prime} / 2\right)\right]^{2}}}{\Delta t^{\prime}}
$$

where $x_{i}(t)$ and $y_{i}(t)$ are the $\mathrm{x}$ and $\mathrm{y}$ coordinates of pedestrian $\mathrm{i}$ at time $\mathrm{t}$ and $\Delta t^{\prime}=0.4 \mathrm{~s}$ is used in this paper.

The average velocity $\mathrm{v}(t)$ is calculated by equation (2):

$$
\mathrm{v}(t)=\frac{\sum_{i}\left\|\bar{v}_{l}(t)\right\|}{N(t)}
$$


The global density $\rho$ is calculated by equation (3):

$$
\rho=\frac{N}{A}
$$

where $\mathrm{N}$ is pedestrian number in measurement area, $\mathrm{A}=28.26 \mathrm{~m}^{2}$ is the size of the measurement area.

The walk path is calculate by equation (4):

$$
\mathrm{S}=\sum_{i=t_{O}}^{t_{D}} \sqrt{\left(x_{t=i+1}-x_{t=i}\right)^{2}+\left(y_{t=i+1}-y_{t=i}\right)^{2}}
$$

where $t_{O}$ and $t_{D}$ is the origin and destination time of pedestrian, $x_{t=i}$ and $y_{t=i}$ are the $\mathrm{x}$ and $\mathrm{y}$ coordinate of pedestrian at time i, $x_{t=i+1}$ and $y_{t=i+1}$ are the $\mathrm{x}, \mathrm{y}$ coordinate of pedestrian at time $\mathrm{i}+1$.

\section{Results and Discussion}

\subsection{The average speed of pedestrians}

To investigate pedestrian speed in different scenarios, we calculate average speed of each pedestrian by equation 1 and 2 and results are shown in figure 3.

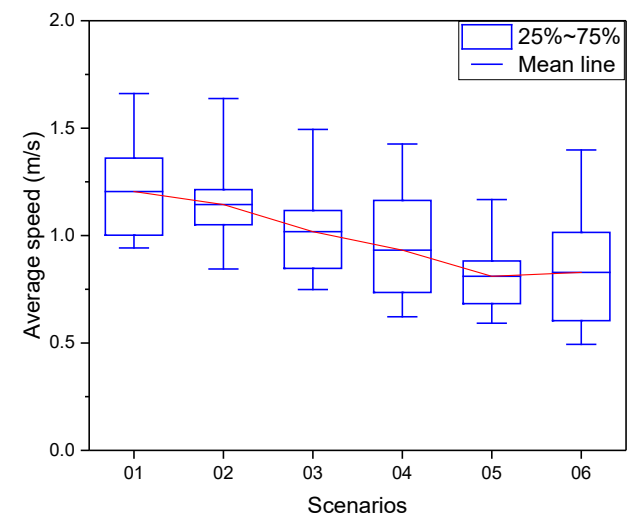

(a)

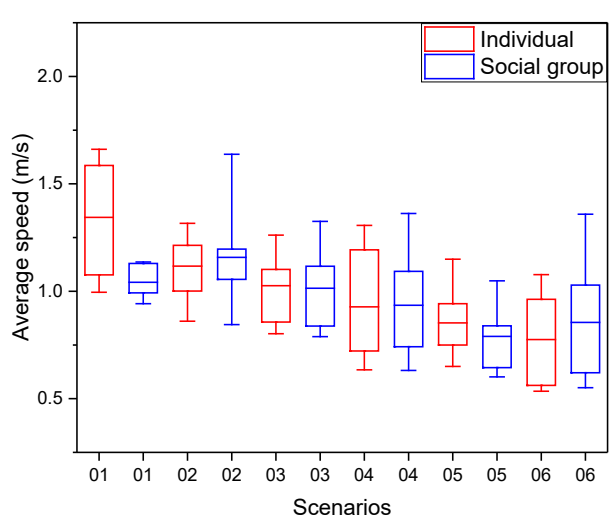

(b)

Fig 3. The average speed of (a) different scenarios and (b) of social group and individual.

As shown in Figure 3(a), with the increasing number of pedestrians in the experiment, the average speed decreases. Since the probability of collision between pedestrians increases with the increasing density in the central area, most of pedestrians have to decrease the walking speed to avoid the conflict with others.

The average speeds of individuals and social groups in different runs are shown in Fig 3. The speed of individual is not always higher than that of social groups. This is contrary to the findings of [7,8] who found that individuals are more flexible than social groups and the latter have a negative influence on the speed of crowds. The influence of social group on pedestrian speed should be deeply investigated.

\subsection{The walk path of pedestrians}

Fig 4 shows the length of the walk path of pedestrians in movement, which increases with pedestrian number. Pedestrians need to walk further to get their destination when there are more pedestrians. Fig 4 (b) shows walk path of social group and individual in different runs. We notice that the walk path of individual is larger than the walk path of social group in each scenario. This conclusion is coincide with the result of Gorrini [8] and Zhao [9]. Due to the flexibility of individual, the walk path of individual is larger than that of social group. 


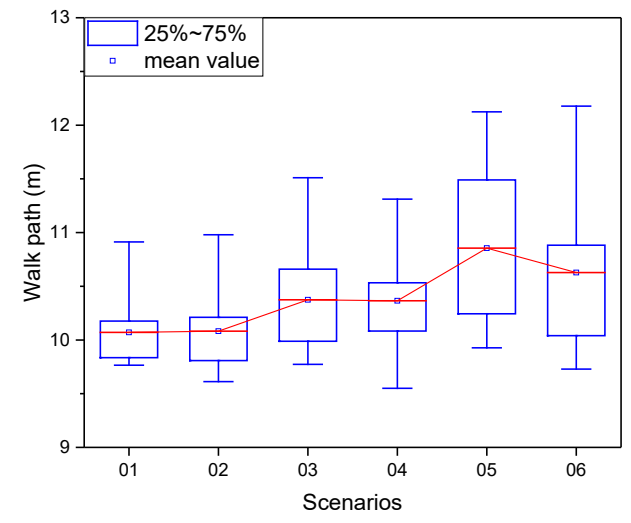

(a)

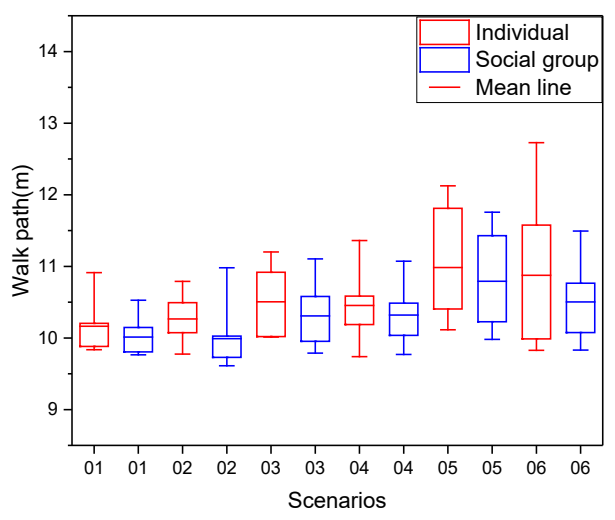

(b)

Fig 4. The walk path (a) in different scenarios and (b) of social group and individual.

\subsection{Strategies of pedestrians}

By analyzing the factors influencing the speed and movement time further, we find that pedestrians adopt different strategies in the process of multi-directional movement. To avoid collisions with others, pedestrians mainly adopt three different strategies in the video recordings: a) decelerating the speed, $b$ ) waiting in situ and letting others go first, c) making a detour. We use pedestrian speed and DC value to classify these strategies into six strategies: 1) Straight - Movement in Steady speed (S-MS), 2) Straight -Movement with Deceleration (S-MD), 3) Straight - Movement with Waiting (S-MW), 4) Curve Movement in Steady speed (C-MS), 5) Curve - Movement with Deceleration (C-MD), 6) Curve Movement with Waiting (C-MW).

We define a Detour Coefficient (DC) value to represent the detour probability of pedestrians during the movement. The DC value is defined as:

$\mathrm{DC}=\left(\frac{\sum_{t=t_{O}}^{t_{D}} \sqrt{\left(x_{t+1}-x_{t}\right)^{2}+\left(y_{t+1}-y_{t}\right)^{2}}}{\sqrt{\left(x_{D}-x_{O}\right)^{2}+\left(y_{D}-y_{O}\right)^{2}}}-1\right) \times 100 \%$

where $x_{t}$ and $y_{t}$ are $\mathrm{x}$ and $\mathrm{y}$ coordinates of pedestrians at time $\mathrm{t}, x_{t+1}$ and $y_{t+1}$ are $\mathrm{x}$ and $\mathrm{y}$ coordinate of pedestrians at time $\mathrm{t}+1, x_{O}$ and $y_{O}$ are $\mathrm{x}$ and $\mathrm{y}$ coordinates of pedestrians at origin position, $x_{D}$ and $y_{D}$ are $\mathrm{x}$ and $\mathrm{y}$ coordinates of pedestrians at destination positions.

During the experiment, the scenario can be approximately divided into two areas where pedestrians show different movement states: 1) the free movement state area: pedestrians move freely and less influenced by others in this area. This area contains two processes, a) at the beginning of the experiment, the self-accelerating process of the pedestrian from standstill to movement, b) at the end of the experiment, the self-deceleration process of the pedestrian from movement to standstill. 2) Congested movement area: congestion occurs in this area due to the accumulation of pedestrians and their speeds are influenced by each other. To facilitate the calculation, we divide the experiment scenario into two parts. Area 1 is a circle with the radius of $3 \mathrm{~m}$ and locates at the center of the experimental site. Moreover, area 1 is the rest of experimental site. From data analysis, we find that there is no difference in speed and movement time between pedestrians in area 1 . Here we focus on the movement of pedestrian in area 2.

Under normal condition, pedestrian will choose the shortest path in movement $[13,14]$. However, under certain circumstances, such as crowded pedestrians, they will make a detour to reach their destination quickly in order to avoid conflicts with other pedestrians and waiting others due to the congestion. Firstly, we divide pedestrians into two categories based on the DC value: 1) DC $<5 \%$ straight movement 2) DC $>5 \%$ curve movement (detour). We find that in the 166 groups (an individual is regarded as one group), 55.4\% (92 groups) move along straight and 44.6\% (74 groups) along curves. This 
is consistent with the shortest path principle in our normal life that most of people prefer to move straight. After manual verification, the $5 \% \mathrm{DC}$ value is used as a standard to divide pedestrians into straight movement and curve movement, the accuracy of the detour criterion is as high as $90 \%$.

Secondly, we divide the pedestrians into three classes according to the speed variation ratio. 1) Movement in Steady speed (MS): the speed variation ratio is less than 25\%. 2) Movement with Deceleration (MD): the speed variation ratio is larger than $25 \%$. The minimal speed is larger than $0.3 \mathrm{~m} / \mathrm{s}$. 3) Movement with Waiting (MW): the speed variation ratio is larger than $25 \%$. The minimal speed is smaller than $0.3 \mathrm{~m} / \mathrm{s}$. In this paper, we think the pedestrian is waiting for others when the speed is less than $0.3 \mathrm{~m} / \mathrm{s}$.

The classify basis and proportion of different strategies are shown in table 2 .

Table 2: The classify basis and proportion of different strategies.

\begin{tabular}{|c|c|c|c|c|}
\hline Strategies & DC value & $\begin{array}{l}\text { Speed variation ratio } \\
\frac{\left(v_{\max }-v_{\min }\right)}{v_{\max }}\end{array}$ & $v_{\min }(\mathrm{m} / \mathrm{s})$ & Proportion \\
\hline $\begin{array}{c}\text { Straight - Movement in } \\
\text { Steady speed } \\
\text { (S-MS) }\end{array}$ & $<5 \%$ & $<25 \%$ & $>0.3$ & $2.4 \%$ \\
\hline $\begin{array}{l}\text { Straight }- \text { Movement } \\
\text { with Deceleration } \\
\text { (S-MD) }\end{array}$ & $<5 \%$ & $>25 \%$ & $>0.3$ & $18.1 \%$ \\
\hline $\begin{array}{l}\text { Straight - Movement } \\
\text { with Waiting } \\
\text { (S-MW) }\end{array}$ & $<5 \%$ & $>25 \%$ & $<0.3$ & $34.9 \%$ \\
\hline $\begin{array}{c}\text { Curve - Movement in } \\
\text { Steady speed } \\
\text { (C-MS) }\end{array}$ & $>5 \%$ & $<25 \%$ & $>0.3$ & $1.8 \%$ \\
\hline $\begin{array}{l}\text { Curve - Movement } \\
\text { with Deceleration } \\
\text { (C-MD) }\end{array}$ & $>5 \%$ & $>25 \%$ & $>0.3$ & $22.9 \%$ \\
\hline $\begin{array}{l}\text { Curve - Movement } \\
\text { with Waiting } \\
\text { (C-MW) }\end{array}$ & $>5 \%$ & $>25 \%$ & $<0.3$ & $19.9 \%$ \\
\hline
\end{tabular}

We plot average speed, density and movement time of different strategies in figure 6 .

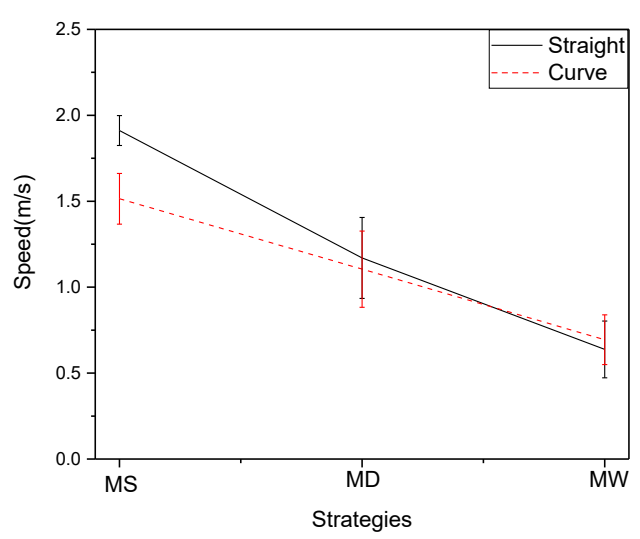

(a)

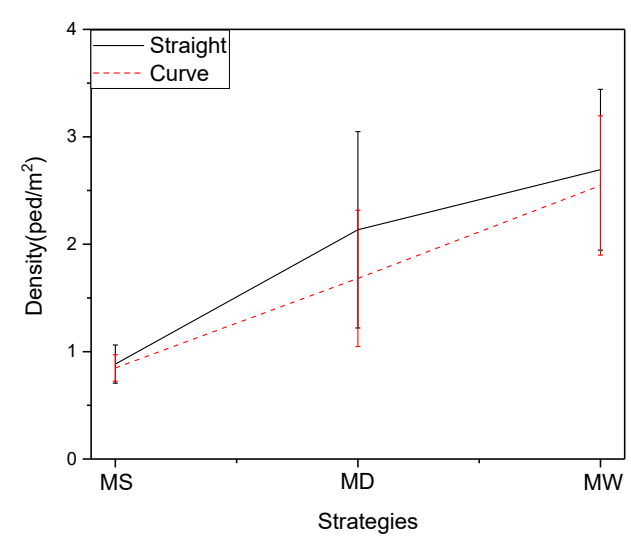

(b) 


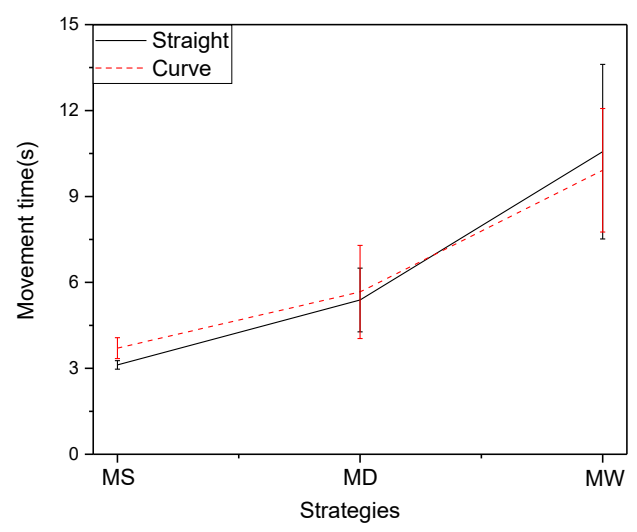

(c)

Fig. 6: (a) the average speed (b) average density and(c) movement time of different strategies.

As can be seen from Fig 6, in six strategies, the MS strategy leads to a higher speed, lower density than and the shortest movement time during the movement. However, this strategy is only observed when the number of participants is small. When the number of pedestrian increases, there is no enough space for them to get through the congested areas in high speed and thus the MS strategy does not work anymore. Due to the change of directions in movement along curve, pedestrian speed of C-MS strategy is smaller than that of S-MS. Owing to the increase of the movement path, the movement time is slightly larger than that of S-MS. However, the density of pedestrians who select the straight movement strategy is larger than that of curve movement strategy. The detour strategy keeps pedestrians away from crowded areas, which results in a lower density and a lower probability of collision with other pedestrians. There is little difference in speed and movement time between S-MD strategy and C-MD strategy. However, the density under C-MD strategy is obviously lower than that under S-MD strategy. It shows that detour strategy is beneficial to keep a certain distance with other pedestrians and to avoid overcrowding between pedestrians. Compared with S-MW strategy, under C-MW strategy pedestrian has higher speed, lower density and shorter movement time. Therefore, curve strategy (detour) is beneficial to improve the movement efficiency in our experiment.

\section{Conclusion}

We conducted a series of experiments with different number of pedestrians to investigate the difference between individuals and social groups in multidirectional crowd movement. We analyse the trajectories, speed and movement time of pedestrians. The results indicate that the speed difference between individual and social group changes based on different conditions. The length of pedestrian walk path increases with the pedestrian density. Moreover, the walk path of social group is shorter than that of individual. Social group do have influence on pedestrian dynamics. We find different strategies for pedestrians to reach their destinations and the speed, density and movement time for different strategies are different. The experiment shows that detour is beneficial in multidirectional movement.

\section{Acknowledgements}

The authors acknowledge the foundation support from the National Natural Science Foundation of China (Grant No. 71704168), from Anhui Provincial Natural Science Foundation (Grant No. 1808085MG217) and the Fundamental Research Funds for the Central Universities (Grant No. WK2320000040).

\section{References}


[1] M. L. Federici, A. Gorrini, L. Manenti, and G. Vizzari, "Data collection for modeling and simulation: case study at the university of milan-bicocca," in International Conference on Cellular Automata, 2012, pp. 699-708: Springer.

[2] C. H. Jazwinski and C. H. Walcheski, "At the mall with children: Group size and pedestrian economy of movement," Environment and Behavior, vol. 43, no. 3, pp. 363-386, 2011.

[3] M. Moussaïd, N. Perozo, S. Garnier, D. Helbing, and G. Theraulaz, "The walking behaviour of pedestrian social groups and its impact on crowd dynamics," PloS one, vol. 5, no. 4, p. e10047, 2010.

[4] M. Schultz, C. Schulz, and H. Fricke, "Passenger dynamics at airport terminal environment," in Pedestrian and Evacuation Dynamics 2008: Springer, 2010, pp. 381-396.

[5] F. Zanlungo, D. Brščić, and T. Kanda, "Spatial-size scaling of pedestrian groups under growing density conditions," Physical Review E, vol. 91, no. 6, p. 062810, 2015.

[6] X. Li, S. Xiong, P. Duan, S. Zheng, B. Li, and M. Liu, "A study on the dynamic spatial-temporal trajectory features of pedestrian small group," in Dependable Computing and Internet of Things (DCIT), 2015 2nd International Symposium on, 2015, pp. 112-116: IEEE.

[7] A. Gorrini, S. Bandini, and M. Sarvi, "Group dynamics in pedestrian crowds: Estimating proxemic behavior," Transportation Research Record: Journal of the Transportation Research Board, no. 2421, pp. 51-56, 2014.

[8] A. Gorrini, S. Bandini, and G. Vizzari, "Empirical investigation on pedestrian crowd dynamics and grouping," in Traffic and Granular Flow'13: Springer, 2015, pp. 83-91.

[9] P. Zhao, L. Sun, L. Cui, W. Luo, and Y. Ding, "The walking behaviours of pedestrian social group in the corridor of subway station," in Proceedings of the 2016 Pedestrian and Evacuation Dynamics Conference, 2016.

[10] J. Wagnild and C. M. Wall-Scheffler, "Energetic consequences of human sociality: walking speed choices among friendly dyads," PloS one, vol. 8, no. 10, p. e76576, 2013.

[11] J. R. Dyer et al., "Consensus decision making in human crowds," vol. 75, no. 2, pp. 461-470, 2008.

[12] M. Boltes, A. Seyfried, B. Steffen, and A. Schadschneider, "Automatic extraction of pedestrian trajectories from video recordings," in Pedestrian and Evacuation Dynamics 2008: Springer, 2010, pp. 43-54.

[13] Y. Guy, "Pedestrian route choice in central Jerusalem," Department of Geography, Ben-Gurion University of The Negev, Beer Sheva (in Hebrew), 1987.

[14] P. Seneviratne and J. Morrall, "Analysis of factors affecting the choice of route of pedestrians," Transportation Planning and Technology, vol. 10, no. 2, pp. 147-159, 1985. 